\title{
Estado nutricional de crianças de descendência haitiana e suas características demográficas, socioeconômicas e de saúde em Cuiabá-MT, Brasil
}

\author{
Nutritional status of children of Haitian descent and their \\ demographic, socioeconomic and health characteristics in Cuiabá, \\ State of Mato Grosso (MT), Brazil
}

Delma Riane Rebouças Batista (https://orcid.org/0000-0003-0113-6463) ${ }^{1}$

Paulo Rogério Melo Rodrigues (https://orcid.org/0000-0002-4213-0685) ${ }^{2}$

Amanda Moura Souza (https://orcid.org/0000-0002-8962-798X) ${ }^{3}$

Rosely Sichieri (https://orcid.org/0000-0001-5286-5354) ${ }^{4}$

Ana Paula Muraro (https://orcid.org/0000-0001-6237-1673) ${ }^{1}$

${ }^{1}$ Instituto de Saúde Coletiva, Departamento de Saúde Coletiva, Universidade Federal de Mato Grosso (UFMT). Av. Fernando Corrêa da Costa 2367, Boa Esperança. 78060900 Cuiabá MT Brasil. muraroap@gmail.com ${ }^{2}$ Departamento de Alimentos e Nutrição, Faculdade de Nutrição, UFMT. Cuiabá MT Brasil.

${ }^{3}$ Instituto de Estudos em Saúde Coletiva, Universidade Federal do Rio de Janeiro. Rio de Janeiro RJ Brasil.

${ }^{4}$ Instituto de Medicina Social, Universidade do Estado do Rio de Janeiro. Rio de Janeiro RJ Brasil.

\begin{abstract}
The scope of this study was to analyze the distribution of anthropometric variables according to demographic, socioeconomic, and health characteristics among children of Haitian descent between 0 and 5 years of age living in Cuiabá-MT. It consists of a cross-sectional and census-based study with data collected between August 2016 and February 2017. The dependent variables were body mass index (BMI) and height-for-age z-score, using Student's t-Test and ANOVA in the statistical analysis. A total of 67 children, $73.2 \%$ to 24 months of age and $34.3 \%$ being male, were evaluated. There was a significant proportion of families with per capita income of less than 1/4 of the minimum wage (65.6\%), with no home monitoring for the family health strategy (88.1\%), and long working hours for mothers (43.3\%). The onset of prenatal care until the third month of pregnancy and female sex were associated with higher BMI averages. For heightfor-age, an association with lower mean values was found for households with more than one resident per room, higher age range of the child, and child-care in daycare centers. Thus, although few factors have an association with the anthropometric indications evaluated, it is necessary to consider the precarious socioeconomic conditions in which these children find themselves.
\end{abstract}

Key words Child health, Emigration and immigration, Nutritional status
Resumo Oobjetivo deste estudo foi analisar a distribuição das variáveis antropométricas de acordo com características demográficas, socioeconômicas e de saúde em crianças, de zero a cinco anos, de descendência haitiana residentes em Cuiabá-MT, Brasil. Consiste em estudo transversal, com dados coletados entre agosto/2016 e fevereiro/2017. As variáveis dependentes foram o indice de massa corporal (IMC) e a estatura/idade em escore- $z$, sendo utilizado o Teste t de Student e ANOVA na análise estatística. Foram avaliadas 67 crianças, $73,2 \%$ com até 24 meses de idade e $34,3 \%$ do sexo masculino. Observou-se expressiva proporção de famílias com renda per capita inferior a 1/4 de salário minimo (65,6\%), sem acompanhamento domiciliar pela Estratégia Saúde da Família $(88,1 \%)$ e elevada carga horária de trabalho das mães (43,3\%). O início do pré-natal até o terceiro mês de gestação e sexo feminino foram associados a maiores médias de IMC. Quanto a estatural idade, foi identificada associação com menores médias para domicílios com mais de um morador por cômodo, faixa etária mais elevada da criança e cuidado da criança em creches. Apesar de poucos fatores apresentaram associação com os indicadores antropométricos avaliados, é necessário atentar-se para as condiçâes socioeconômicas precárias nas quais estas crianças estão inseridas.

Palavras-chave Saúde da criança, Emigração e imigração, Estado nutricional 


\section{Introdução}

A infância compreende um processo de crescimento e desenvolvimento complexo e pode ser influenciado por fatores multivariados. Os indicadores antropométricos nessa fase da vida podem evidenciar situações de risco, auxiliando no diagnóstico de saúde, além de subsidiar planejamento de ações de promoção de saúde e prevenção de doenças que mais afetam às crianças' ${ }^{1}$. Para avaliação adequada do crescimento e desenvolvimento de uma criança é necessário conhecer também seu contexto familiar e socioeconômi$\mathrm{co}^{2}$. Um contexto familiar com baixas condições socioeconômicas pode interferir negativamente no crescimento durante a infância, no entanto, se as famílias nesta condição forem acompanhadas e orientadas adequadamente, é possível minimizar possíveis danos ${ }^{3}$. Condições socioeconômicas inadequadas estão presentes no contexto de muitas famílias imigrantes, com possível reflexo sobre a saúde de suas crianças.

O estado nutricional das crianças haitianas está entre os mais deficientes da América Latina e região do Caribe ${ }^{4}$, mesmo com queda na taxa de desnutrição infantil de 28,5\% em 2005-06 para $22,2 \%$ em 2012 entre as crianças haitianas de zero a cinco anos de idade incompletos ${ }^{5}$. Apesar dessa melhoria, a Organização das Nações Unidas verificou que o Haiti ainda possuía a maior prevalência de subalimentação do planeta em 2016 (53,4\%), apresentando também a menor prevalência de sobrepeso na população geral entre os países da América Latina e Caribe (38,5\%) e apenas 3,6\% das crianças haitianas menores de cinco anos apresentam sobrepeso ${ }^{4}$.

A migração de haitianos é uma realidade há décadas, mas a partir de 2010, o Brasil se destacou como um dos principais países de destino, em decorrência de diversos motivos, entre os quais se destacam a Copa do Mundo FIFA, a maior exigência para entrada em outros países ${ }^{6} \mathrm{e}$ a proximidade com os brasileiros, que lideram a Missão das Nações Unidas para Estabilização do Haiti (MINUSTAH) desde 20047 . Estima-se que o número de haitianos no Brasil, em 2015, era de aproximadamente 55.000 imigrantes $^{8}$, sendo que, no mesmo ano, Cuiabá, capital do Estado de Mato Grosso (MT), destacou-se como o quarto município do país em número de haitianos registrados no mercado formal de trabalho'. Inicialmente, o contexto migratório da população haitiana era formado em sua maioria por homens, no entanto, foi observada elevação no fluxo de mulheres e crianças imigrantes no Brasil ${ }^{10}$.
Não foram encontrados dados censitários no Brasil que estime a população de crianças haitianas ou de descendência direta. Em Mato Grosso, foram registrados no Sistema de Informação de Nascimento do Ministério da Saúde (SINASC) 139 nascimentos de filhos de mulheres de nacionalidade haitiana entre os anos de 2013 e $2015^{11}$, sendo que destes, $95(68,3 \%)$ ocorreram em Cuiabán ${ }^{12}$.

$\mathrm{Na}$ análise desses registros quanto às características socioeconômicas e de atenção à saúde, comparadas às mães brasileiras, verificou-se que as mães haitianas apresentaram menor escolaridade, menor proporção do número de consultas pré-natais e maior prevalência de nascidos vivos com baixo peso ao nascer ${ }^{11}$.

$\mathrm{Na}$ literatura pesquisada, não foram encontrados estudos no Brasil que avaliassem as características socioeconômicas e de atenção a saúde das famílias com crianças de descendência haitiana direta, sejam elas de nacionalidade brasileiras ou não. Conhecer o perfil desse grupo populacional possibilitaria o planejamento de assistência adequada a essa população, o que é essencial para minimizar possíveis agravos a sua saúde e, consequentemente, reduzir a demanda e a sobrecarga ao sistema de saúde local.

Nesse sentido, o presente trabalho tem por objetivo analisar as características demográficas, socioeconômicas, obstétricas e de cuidado em saúde e sua associação com indicadores antropométricos de crianças haitianas, ou de descendência direta, com idade entre zero e cinco anos, residentes em Cuiabá-MT.

\section{Métodos}

Censo de crianças filhas de mulheres haitianas residentes em Cuiabá, capital do estado de Mato Grosso, com base no registro do SINASC e com o apoio do registro do Centro de Pastoral para Migrantes de Cuiabá. A população de estudo consistiu em crianças de ambos os sexos, com idades entre zero e cinco anos, nascidas ou não em território brasileiro. A coleta de dados foi realizada nos domicílios ou espaços compartilhados (igrejas, creches e Centro de Pastoral do Migrante) por imigrantes no município.

Buscou-se avaliar a totalidade das crianças que vivem no município, visto que não há dados exatos do número de crianças de descendência direta de mulheres imigrantes haitianas no referido município. Foram considerados os registros de nascidos disponíveis no SINASC de filhos de 
mulheres de nacionalidade haitianas residentes em Mato Grosso nos anos de 2013, 2014 e 2015. Os dados utilizados foram fornecidos pela Secretaria Estadual de Saúde de Mato Grosso.

Durante a coleta de dados, as mães e as crianças foram avaliadas em duas etapas: entrevista (mãe) e avaliação antropométrica (mãe e criança), ambos no mesmo momento. A entrevista foi realizada por um entrevistador bilíngue (Creole haitiano/Português) e a avaliação antropométrica por avaliador treinado, logo após a entrevista. O questionário foi constituído de questões já utilizadas em inquérito anterior com haitianos na capital mato-grossense em 2014/2015 ${ }^{13,14}$, com estudo piloto realizado com um grupo de indivíduos não incluídos na população de estudo. Além disso, grande parte das questões utilizadas neste estudo são informações rotineiramente coletadas em registros oficinais como SINASC (por exemplo, as informações obstétricas da mãe e as relativas ao nascimento da criança). O questionário específico utilizado para esta pesquisa foi testado com uma mãe haitiana em agosto de 2016 com o objetivo de verificar e corrigir possíveis falhas durante a coleta de dados. Os dados foram coletados entre os meses de agosto de 2016 e fevereiro de 2017.

A avaliação do peso das crianças menores de dois anos foi realizada com a criança nua, em balança pediátrica com capacidade máxima de $20 \mathrm{~kg}$, com variação de $10 \mathrm{~g}$. No presente estudo foi utilizada balança pediátrica digital, marca Tanita, modelo BD-584. Para a medida do comprimento, foi utilizada Estadiômetro Infantil Portátil (Marca Seca, modelo 417), com variação de $1 \mathrm{~mm}$, avaliando a criança em posição supina e com leito horizontal completo, sobre uma superfície firme e com os joelhos esticados. Para avaliação do comprimento de criança nessa faixa etária, foi necessário o auxílio de uma segunda pessoa, comumente a mãe, para garantir a medição adequada da criança.

$O$ peso das crianças com idade superior a dois anos e de suas mães foram avaliados por meio de balança digital, marca OMRON, modelo HBF 510W, com capacidade máxima de $150 \mathrm{~kg}$ e com precisão de $100 \mathrm{~g}$. Para a mensuração da estatura da criança de dois anos ou mais de idade e de suas mães foi utilizado antropômetro portátil da marca Sanny, com variação de $1 \mathrm{~mm}$ e extensão de até $210 \mathrm{~cm}$. Estas medidas foram realizadas com o indivíduo descalço, usando roupas leves, sem adornos na cabeça e em posição ortostática, com a cabeça posicionada respeitando-se o plano de Frankfurt. Foram realizadas duas mensura- ções de estatura, admitindo-se variação máxima de $0,5 \mathrm{~cm}$, considerando a média para análise. As medidas de peso e estatura foram mensuradas conforme técnica descrita por Araújo ${ }^{15}$.

Calculou-se o índice de massa corporal (IMC) para idade e a estatura para idade, em escore-z, de acordo com as curvas da Organização Mundial de Saúde ${ }^{16}$, utilizando o programa WHO Anthro, versão 3.2.2.

As variáveis sociodemográficas e econômicas avaliadas foram: faixa etária materna ( $\leq 25$ anos, entre 26 e 35 anos, entre 36 e 45 anos); estado civil materno (casada/união estável, solteira/ viúva); escolaridade materna (em anos de estudo: $<8$ anos, $\geq 8$ anos); tempo de residência no Brasil ( $\leq 24$ meses, $>24$ meses); compreensão de português (muito pouco ou pouco, razoavelmente, bem ou muito bem); trabalho atual no Brasil (sim, não); carga horária de trabalho ( $\leq$ 44 horas/semana, $>44$ horas/semana); número de moradores/cômodo ( $\leq 1$ pessoa/cômodo, $>1$ pessoa/cômodo); renda familiar mensal per capita (em reais, segundo fração do salário mínimo: $<234,25$, entre 234,25 e 468,50, entre 468,50 e 937,00 ); tempo de permanência no domicílio (< 12 meses, $\geq 12$ meses); presença de unidade de saúde no bairro (sim, não); visita da Estratégia Saúde da Família (ESF) nos últimos 12 meses (pelo menos uma vez, nunca recebeu).

Quanto aos dados obstétricos antropométricos maternos e relacionados ao cuidado da criança, avaliou-se: idade da primeira gestação ( $\leq$ 20 anos, entre 21 e 29 anos, $\geq 30$ anos); aborto ( $\operatorname{sim}$, não); histórico gestacional ( $\leq 3$ gestações, $>3$ gestações); IMC materno atual (eutrofia, sobrepeso, obeso, segundo a Organização Mundial de Saúde (OMS) ${ }^{17}$; início do pré-natal (até $3^{\circ}$ mês de gestação, após $3^{\circ}$ mês); número de consultas pré-natal ( $<6$ consultas, $\geq 6$ consultas); tipo de parto (vaginal, cesárea); nasceu no Brasil (sim, não); sexo da criança (masculino, feminino); peso ao nascer (em gramas: $\geq 2.500 \mathrm{~g},<2.500 \mathrm{~g}$ ); idade da criança (em meses: $\leq 12$ meses, entre 12 e 24 meses, > 24 meses); cuidado diário da criança (diurno: creche, mãe, outros); acompanhamento em estabelecimento de saúde ( $\operatorname{sim}$, não); tempo de aleitamento materno exclusivo - AME ( $<3$ meses, entre 3 e 6 meses, $\geq 6$ meses, ainda amamenta) e hospitalização alguma vez na vida (sim, não).

Foi realizada dupla digitação dos questionários por meio do software Epi Info 7 e os dados foram analisados com o auxílio do programa estatístico SPSS, versão 17. Foram calculadas as distribuições de frequências, bem com as medi- 
das de tendência central e dispersão das variáveis de interesse.

Os desfechos IMC para idade e Estatura para idade em escore-z foram testados quanto à natureza de sua distribuição pelo teste de Kolmogorov-Smirnov, apresentando distribuição normal. $\mathrm{Na}$ análise bivariada, foram utilizados o Teste $t$ de Student e ANOVA para testar diferenças das médias entre os grupos. Considerando que o dimensionamento de amostras a priori não foi factível para o presente estudo, justifica-se o cálculo amostral a posterior ${ }^{18}$, que foi realizado segundo o proposto por Luiz e Magnini ${ }^{19}$ para diferença entre médias. Desta forma, somente diferenças igual ou maior do que 0,42 teriam a possibilidade de mostrar diferenças estatisticamente significativas com um poder de $80 \%$ e um erro de 0,05 .

O estudo foi aprovado pelo Comitê de Ética do Hospital Júlio Muller da Universidade Federal de Mato Grosso. As mães assinaram um Termo de Consentimento Livre e Esclarecido. O projeto recebeu financiamento pela Fundação de Amparo à Pesquisa do Estado de Mato Grosso e pelo Conselho Nacional de Desenvolvimento Científico e Tecnológico.

\section{Resultados}

Foram avaliadas 67 crianças, sendo $73,2 \%$ com até 24 meses de idade e $34,3 \%$ do sexo masculino. Apenas duas crianças apresentaram escore z IMC para idade menor que -2 (magreza) e nenhuma criança foi classificada com excesso de peso (escore-z $>2$ ). Quanto ao índice de estatura para idade, nenhuma criança foi classificada como baixa estatura para idade (escore- $\mathrm{z}<-2$ ).

Com relação às características maternas, $70,1 \%$ tinham entre 26 e 35 anos de idade, $83,6 \%$ informaram estarem casadas ou em união estável e $73,1 \%$ tinham 8 anos ou mais de estudo. Mais da metade das mulheres informaram que residiam no Brasil há mais de 2 anos (59,7\%), 70,1\% disseram entender razoavelmente, bem ou muito bem o português, $47,8 \%$ estavam trabalhando no período da coleta, sendo que, dentre estas, $65,6 \%$ cumpriam carga horária semanal superior a 44 horas. Não foram verificadas diferenças estatisticamente significativas do IMC para idade e estatura segundo as características sociodemográficas das mães (Tabela 1).

Quando analisado o IMC para idade segundo as características do ambiente domiciliar e a cobertura da ESF (Tabela 2), identificou-se que $32,8 \%$ dos domicílios possuíam mais que uma pessoa por cômodo, sendo que 43,3\% das entrevistadas relataram renda per capita inferior a $\mathrm{R} \$$ 234,25 (1/4 do salário mínimo atual). Apesar de $47,8 \%$ relatarem que residiam no mesmo domicílio há mais de 1 ano, $88,1 \%$ referiram nunca ter recebido visita de integrantes da ESF. Foram observadas menores médias de estatura para idade entre crianças de famílias com mais de um morador por cômodo (Tabela 2).

Em relação às características antropométricas e obstétricas das mulheres entrevistadas (Tabela 3) $22,4 \%$ tiveram sua primeira gestação com idade igual ou inferior a 20 anos, $23,9 \%$ já sofreram algum aborto, sendo que apenas uma referiu aborto provocado e $26,9 \%$ informaram histórico de três ou mais gestações. Quanto ao IMC materno atual, $68,6 \%$ das mulheres foram classificadas como sobrepeso ou obesidade. Nenhuma dessas variáveis apresentou diferença estatisticamente significativa quanto ao IMC para idade e estatura por idade da criança.

Ao informar sobre a gestação e o acompanhamento pré-natal referente à criança avaliada (Tabela 4), 91,0\% iniciaram o acompanhamento pré-natal até o terceiro mês gestacional (independente do país que residiam), apresentando maior média de escore-z de IMC para idade, quando comparadas àquelas que iniciaram o pré-natal após o terceiro mês de gestação ( $\mathrm{p}$-valor $<0,01$ ). Quanto à quantidade de consultas pré-natal realizadas, $77,6 \%$ realizaram seis ou mais consultas e a maioria dos nascimentos foi por parto vaginal $(62,7 \%)$, sendo que todas as entrevistadas relataram a realização do parto hospitalar. Dentre as crianças avaliadas, $34,3 \%$ são do sexo masculino, apresentando menor média de escore-z de IMC para idade quando comparada ao sexo feminino e, $10,4 \%$ apresentaram peso inferior a 2.500 gramas ao nascer. Observou-se ainda menores médias e estatura para idade entre as crianças em maiores faixas etárias e aquelas que frequentavam creche diariamente.

A maioria das crianças avaliadas nasceu no Brasil $(89,6 \%)$, sendo apenas três crianças nascidas no Haiti, três na República Dominicana e uma na Guiana Francesa. Dentre as entrevistadas, que referiram início da gestação no território brasileiro $(73,2 \%), 91,8 \%$ iniciaram o acompanhamento pré-natal até o terceiro mês de gestação e apenas $20,4 \%$ realizaram menos que seis consultas pré-natais. Complicações durante a gestação como diabetes gestacional, doença hipertensiva específica da gravidez e hiperemese foram relatadas por $31,3 \%$ das entrevistadas e 92,5\% dos partos foram cobertos pela rede pública de saúde (dados não apresentados em tabelas). 
Tabela 1. Distribuição dos índices Índice de Massa Corporal-para-idade e estatura-para-idade (média [m] e desvio-padrão [dp]) de crianças até 5 anos de idade, filhas de mulheres haitianas, segundo características sociodemográficas maternas. Cuiabá-MT, 2016-2017.

\begin{tabular}{|c|c|c|c|c|c|c|}
\hline & \multicolumn{2}{|c|}{ Geral } & \multicolumn{2}{|c|}{$\begin{array}{l}\text { IMC-para-idade } \\
\text { (escore-z) }\end{array}$} & \multicolumn{2}{|c|}{$\begin{array}{c}\text { Estatura-para-idade } \\
\text { (escore-z) }\end{array}$} \\
\hline & $\mathbf{N}$ & $(\%)$ & $\mathbf{m}$ & $(d p)$ & $\mathbf{m}$ & $(d p)$ \\
\hline \multicolumn{7}{|l|}{ Faixa etária da mãe (anos) } \\
\hline$\leq 25$ & 05 & $(7,5)$ & $-0,30$ & $(1,38)$ & 0,61 & $(1,85)$ \\
\hline 26 a 35 & 47 & $(70,1)$ & $-0,14$ & $(1,02)$ & 0,75 & $(1,34)$ \\
\hline 36 a 45 & 15 & $(22,4)$ & $-0,22$ & $(0,90)$ & 0,44 & $(0,98)$ \\
\hline p-valor & & & \multicolumn{2}{|c|}{0,92} & \multicolumn{2}{|c|}{0,19} \\
\hline \multicolumn{7}{|l|}{ Estado civil da mãe } \\
\hline Casada/União estável & 56 & $(83,6)$ & $-0,18$ & $(1,06)$ & 0,47 & $(1,35)$ \\
\hline Solteira/viúva ${ }^{a}$ & 11 & $(16,4)$ & $-0,12$ & $(0,75)$ & 1,16 & $(1,04)$ \\
\hline p-valor ${ }^{c}$ & & & \multicolumn{2}{|c|}{0,86} & \multicolumn{2}{|c|}{0,11} \\
\hline \multicolumn{7}{|l|}{ Escolaridade da mãe } \\
\hline$<8$ anos & 18 & $(26,9)$ & $-0,25$ & $(0,96)$ & 0,32 & $(0,73)$ \\
\hline$\geq 8$ anos & 49 & $(73,1)$ & $-0,14$ & $(1,03)$ & 0,68 & $(1,48)$ \\
\hline p-valor & & & & 0,68 & & 0,33 \\
\hline \multicolumn{7}{|l|}{ Tempo de residência da mãe no Brasil ${ }^{\mathrm{b}}$} \\
\hline$\leq 24$ meses & 26 & $(38,8)$ & $-0,12$ & $(0,88)$ & 0,52 & $(0,98)$ \\
\hline$>24$ meses & 40 & $(59,7)$ & $-0,21$ & $(1,11)$ & 0,64 & $(1,52)$ \\
\hline p-valor & & & \multicolumn{2}{|c|}{0,71} & \multicolumn{2}{|c|}{0,72} \\
\hline \multicolumn{7}{|l|}{ Entende português } \\
\hline Muito Pouco ou pouco & 20 & $(29,9)$ & $-0,32$ & $(0,98)$ & 0,88 & $(1,68)$ \\
\hline Razoavelmente, bem ou muito bem & 47 & $(70,1)$ & $-0,10$ & $(1,02)$ & 0,46 & $(1,14)$ \\
\hline p-valor ${ }^{c}$ & & & \multicolumn{2}{|c|}{0,41} & \multicolumn{2}{|c|}{0,23} \\
\hline \multicolumn{7}{|l|}{ Trabalho atual no Brasil } \\
\hline Sim & 32 & $(47,8)$ & 0,01 & $(0,87)$ & 0,49 & $(1,19)$ \\
\hline Não & 35 & $(52,2)$ & $-0,33$ & $(1,11)$ & 0,67 & $(1,44)$ \\
\hline p-valor ${ }^{c}$ & & & & 0,17 & & 0,59 \\
\hline \multicolumn{7}{|l|}{ Carga horário de trabalho } \\
\hline$\leq 44$ horas/semana & 11 & $(34,4)$ & 0,01 & $(1,02)$ & 0,38 & $(1,22)$ \\
\hline$>44$ horas/semana & 21 & $(65,6)$ & 0,01 & $(0,81)$ & 0,55 & $(1,20)$ \\
\hline p-valor ${ }^{\mathrm{c}}$ & & & \multicolumn{2}{|c|}{0,99} & \multicolumn{2}{|c|}{0,70} \\
\hline
\end{tabular}

${ }^{\mathrm{a}}$ Apenas uma mãe relatou ser viúva; ${ }^{\mathrm{b}}$ Informações faltantes para 1 entrevistada; ${ }^{\mathrm{c}} P$-valor: ANOVA ou Teste $t$ de Student - Apenas diferenças igual ou maior do que 0,42 , teriam a possibilidade de mostrar diferenças estatisticamente significativas com um poder de $80 \%$ e um erro de 0.05 .

A avaliação da situação vacinal não foi registrada em tabela, devido à quantidade de crianças que não teve seus cartões de vacina avaliados na coleta $(\mathrm{n}=28)$, a importante proporção de crianças sem essa informação foi devido a coleta de dados ocorrer em locais como igrejas e casas de outrem, fazendo com que muitas vezes as mães não estivessem com o documento em mãos. Contudo, dentre os cartões de vacina avaliados, $22,4 \%$ apresentavam atraso em alguma dose de vacina que compõe o Calendário Nacional de Vacinação. Algumas crianças estavam em atraso quanto a seis tipos diferentes de vacina $(n=4)$.

\section{Discussão}

O perfil antropométrico, socioeconômico e de atenção à saúde das crianças filhas de mulheres haitianas no Brasil, mostrou que apesar da expressiva proporção de famílias com baixa renda, sem acompanhamento domiciliar pela ESF e elevada carga horária de trabalho das mães, apenas duas crianças apresentaram IMC abaixo do esperado para a idade $(3,0 \%)$ e nenhuma criança foi classificada como baixa estatura. Contudo, foi identificada associação com menores médias para domicílios com mais de um morador por 
Tabela 2. Distribuição dos índices Índice de Massa Corporal-para-idade e estatura-para-idade (média [m] e desvio-padrão [dp]) de crianças até 5 anos de idade, filhas de mulheres haitianas, segundo características do ambiente domiciliar e cobertura da Estratégia Saúde da Família (ESF) dos domicílios. Cuiabá-MT, 2016-2017.

\begin{tabular}{|c|c|c|c|c|c|c|}
\hline & \multicolumn{2}{|c|}{ Geral } & \multicolumn{2}{|c|}{$\begin{array}{l}\text { IMC-para-idade } \\
\text { (escore-z) }\end{array}$} & \multicolumn{2}{|c|}{$\begin{array}{l}\text { Estatura-para- } \\
\text { idade (escore-z) }\end{array}$} \\
\hline & $\mathbf{N}$ & $(\%)$ & $\mathbf{m}$ & $(\mathrm{dp})$ & m & $(d p)$ \\
\hline \multicolumn{7}{|c|}{ Número de moradores/cômodo ${ }^{a}$} \\
\hline$\leq 1$ pessoa/cômodo & 44 & $(65,7)$ & $-0,13$ & $(0,82)$ & 0,80 & $(1,35)$ \\
\hline$>1$ pessoa/cômodo & 22 & $(32,8)$ & $-0,03$ & $(0,91)$ & 0,09 & $(1,16)$ \\
\hline p-valor ${ }^{b}$ & & & \multicolumn{2}{|c|}{0,66} & \multicolumn{2}{|c|}{0,04} \\
\hline \multicolumn{7}{|c|}{ Renda familiar mensal per capita ${ }^{a}$ (em reais) } \\
\hline$<234,25$ & 29 & & $-0,23$ & $(1,23)$ & 0,94 & $(1,64)$ \\
\hline$\geq 234,25$ e $<468,5$ & 27 & & 0,08 & $(0,76)$ & 0,11 & $(0,87)$ \\
\hline$\leq 937,00$ & 11 & & $-0,61$ & $(0,79)$ & 0,81 & $(1,00)$ \\
\hline p-valor ${ }^{\mathrm{b}}$ & & & \multicolumn{2}{|c|}{0,14} & \multicolumn{2}{|c|}{0,05} \\
\hline \multicolumn{7}{|c|}{ Tempo de permanência no domicílio } \\
\hline$<12$ meses & 35 & $(52,2)$ & $-0,17$ & $(1,18)$ & 0,60 & $(1,10)$ \\
\hline$\geq 12$ meses & 32 & $(47,8)$ & $-0,17$ & $(0,81)$ & 0,57 & $(1,55)$ \\
\hline p-valor ${ }^{\mathrm{b}}$ & & & \multicolumn{2}{|c|}{0,99} & \multicolumn{2}{|c|}{0,93} \\
\hline \multicolumn{7}{|c|}{ Presença de Unidade de Saúde no bairro } \\
\hline Sim & 37 & $(55,2)$ & $-0,33$ & $(1,21)$ & 0,57 & $(1,51)$ \\
\hline Não & 30 & $(44,8)$ & 0,03 & $(0,66)$ & 0,59 & $(1,06)$ \\
\hline p-valor ${ }^{b}$ & & & \multicolumn{2}{|c|}{0,12} & \multicolumn{2}{|c|}{0,95} \\
\hline \multicolumn{7}{|c|}{ Visita da ESF nos últimos 12 meses $^{\mathrm{a}}$} \\
\hline Pelo menos uma vez & 07 & $(10,4)$ & 0,31 & $(0,52)$ & 0,45 & $(1,37)$ \\
\hline Nunca recebeu & 59 & $(88,1)$ & $-0,15$ & $(0,87)$ & 0,58 & $(1,33)$ \\
\hline p-valor ${ }^{\mathrm{b}}$ & & & \multicolumn{2}{|c|}{0,18} & \multicolumn{2}{|c|}{0,80} \\
\hline
\end{tabular}

cômodo, faixa etária mais elevada da criança $(>$ 24 meses) e cuidado diário da criança realizado em creches.

No Brasil, em contextos sociais semelhantes, Felisbino-Mendes et al. ${ }^{20}$ avaliaram 1.322 crianças entre zero e 10 anos de idade, residentes no município de Ferros-MG e verificaram que 3,8\% dos participantes apresentaram algum grau de desnutrição e 3,0\% foram classificadas com baixa estatura para idade. Outro estudo envolvendo crianças de um centro educacional da rede pública do Rio de Janeiro, foi observada prevalência de 7,2\% de baixo peso ou risco para baixo peso a partir da avaliação do IMC para idade, bem como $1,6 \%$ de crianças apresentando baixa estatura para idade ${ }^{21}$. Em Mato Grosso, Menegaz et al. ${ }^{22}$, a partir da avaliação da população da área de influência da BR 163 (Alta Floresta, Sinop, Sorriso e Diamantino), observaram a prevalência de 5,0\% de baixo IMC para idade entre as crianças de 2 a 9 anos de idade e 2,9\% com baixa estatura no mesmo grupo etário. Dessa forma, pode-se inferir que o grupo estudado no presente trabalho apresenta quadro semelhante, ou relativamente melhor, ao observado entre crianças brasileiras em situações socioeconômicas comparáveis.

Quando comparada à situação nutricional das crianças nessa mesma faixa etária residentes no Haiti, os resultados verificados neste estudo indicam melhores condições de saúde deste grupo populacional no Brasil. Dados divulgados pela Organização das Nações Unidas ${ }^{4}$ indicaram que no ano de 2016 o país ainda possuía a maior prevalência de subalimentação do planeta $(53,4 \%)$. Quanto à desnutrição infantil, apesar da redução de 40,1\% em 1990 para 21,9\% em 2012, o Haiti ainda ocupava o quinto lugar no ranking de países da América Latina e Caribe com maior índice de desnutrição crônica infantil, ficando atrás apenas da Guatemala, Equador, Nicarágua e Honduras ${ }^{4}$.

No presente estudo, a maioria das mães tinham entre 26 e 35 anos de idade e pelo menos 
Tabela 3. Distribuição dos índices Índice de Massa Corporal-para-idade e estatura-para-idade (média [m] e desvio-padrão [dp]) de crianças até 5 anos de idade, filhas de mulheres haitianas, segundo características antropométricas e obstétricas das mães. Cuiabá-MT, 2016-2017.

\begin{tabular}{|c|c|c|c|c|c|c|}
\hline & \multicolumn{2}{|c|}{ Geral } & \multicolumn{2}{|c|}{$\begin{array}{l}\text { IMC-para-idade } \\
\text { (escore-z) }\end{array}$} & \multicolumn{2}{|c|}{$\begin{array}{c}\text { Estatura-para-idade } \\
\text { (escore-z) }\end{array}$} \\
\hline & $\mathbf{N}$ & $(\%)$ & $\mathbf{m}$ & $(d p)$ & $\mathbf{m}$ & $(d p)$ \\
\hline \multicolumn{7}{|c|}{ Idade da primeira gestação } \\
\hline$\leq 20$ anos & 15 & $(22,4)$ & $-0,21$ & $(0,93)$ & 0,16 & $(1,04)$ \\
\hline 21 a 29 anos & 44 & $(65,7)$ & $-0,18$ & $(1,07)$ & 0,73 & $(1,49)$ \\
\hline$\geq 30$ anos & 08 & $(11,9)$ & 0,01 & $(0,88)$ & 0,55 & $(0,33)$ \\
\hline p-valor ${ }^{\mathrm{b}}$ & & & \multicolumn{2}{|c|}{0,86} & \multicolumn{2}{|c|}{0,35} \\
\hline \multicolumn{7}{|l|}{ Aborto $^{\mathrm{a}}$} \\
\hline Sim & 16 & $(23,9)$ & 0,15 & $(0,72)$ & 0,38 & $(1,16)$ \\
\hline Não & 51 & $(76,1)$ & $-0,27$ & $(1,07)$ & 0,65 & $(1,37)$ \\
\hline p-valor ${ }^{b}$ & & & \multicolumn{2}{|c|}{0,15} & \multicolumn{2}{|c|}{0,48} \\
\hline \multicolumn{7}{|c|}{ Histórico gestacional } \\
\hline$\leq 3$ gestações & 49 & $(73,1)$ & $-0,29$ & $(1,08)$ & 0,66 & $(1,39)$ \\
\hline$>3$ gestações & 18 & $(26,9)$ & 0,16 & $(0,73)$ & 0,38 & $(1,13)$ \\
\hline p-valor ${ }^{\mathrm{b}}$ & & & \multicolumn{2}{|c|}{0,10} & \multicolumn{2}{|c|}{0,46} \\
\hline \multicolumn{7}{|c|}{ IMC materno atual } \\
\hline Eutrófico & 21 & $(31,4)$ & $-0,02$ & $(0,78)$ & 0,78 & $(1,29)$ \\
\hline Sobrepeso & 22 & $(32,8)$ & $-0,51$ & $(1,21)$ & 0,12 & $(0,91)$ \\
\hline Obeso & 24 & $(35,8)$ & 0,01 & $(0,95)$ & 0,84 & $(1,58)$ \\
\hline $\mathrm{p}$-valor ${ }^{\mathrm{b}}$ & & & \multicolumn{2}{|c|}{0,15} & \multicolumn{2}{|c|}{0,13} \\
\hline
\end{tabular}

8 anos de estudo. Essas características de idade e escolaridade materna devem ser avaliadas considerando as características sociodemográficas das mulheres haitianas, principalmente daquelas que migram para outros países. Em estudo prévio com os registros de nascimento no SINASC do estado de Mato Grosso, observou-se que enquanto $11,9 \%$ das mães haitianas apresentaram escolaridade de até 5 anos de estudo completos, apenas $4,9 \%$ das mulheres brasileiras possuíam esse nível de escolaridade, o que parece indicar dificuldade de acesso à educação entre as mulheres haitianas ${ }^{11}$.

Segundo Joint ${ }^{23}$, no Haiti, o sistema educacional tem por objetivo manter a separação das elites quanto às massas ou classes populares pobres, principalmente devido ao aumento descontrolado do número de escolas particulares que provocou um processo de privatização da educação. No ano de 2008, 83,0\% das escolas no Haiti pertenciam ao sistema privado $^{24}$ dificultando, assim, o acesso a graus mais elevados de escolaridade pela população menos privilegiada. Segundo dados do Ministério do Trabalho e Emprego do Brasil, no ano de 2012 apenas 17,3\% dos haitianos que solicitaram autorização para residência no país possuíam escolaridade entre ensino médio completo e superior ${ }^{24}$ Em Mato Grosso, 11,8\% dos imigrantes haitianos residentes na capital do estado relataram possuir ensino médio completo ou mais, sendo menor proporção de mulheres $(2,7 \%)$ quando comparadas aos homens $(13,7 \%)$ com esse nível de escolaridade ${ }^{13}$.

Quanto aos fatores relacionados à migração, apesar de a maioria das mulheres referir residir no Brasil há mais de dois anos e relatar compreensão razoável, boa ou muito boa da língua portuguesa, não se pode descartar a possibilidade dessas características serem barreiras para o acesso ao sistema de saúde local ${ }^{25}$. Pesquisa realizada no Brasil, com migrantes bolivianos em São Paulo, mostrou associação positiva entre o tempo de residência no Brasil com a utilização de diferentes serviços de saúde, especialmente a atenção primária em saúde, que foi mais utilizada entre aqueles que viviam há mais tempo no país ${ }^{26}$. Em Cuiabá, foi observado que a utilização de serviços de saúde foi duas vezes mais prevalente entre os imigrantes haitianos que referiram falar e compreender bem ou muito bem o português, quando comparados aos que compreendiam muito pouco, porém, ao ser ajustado pelo tempo 
Tabela 4. Distribuição dos índices Índice de Massa Corporal-para-idade e estatura-para-idade (média [m] e desvio-padrão [dp]) de crianças até 5 anos de idade, filhas de mulheres haitianas, segundo características do acompanhamento pré-natal, parto e de cuidados da criança. Cuiabá-MT, 2016-2017.

\begin{tabular}{|c|c|c|c|c|c|c|}
\hline & \multicolumn{2}{|c|}{ Geral } & \multicolumn{2}{|c|}{$\begin{array}{c}\text { IMC-para-idade } \\
\text { (escore-z) }\end{array}$} & \multicolumn{2}{|c|}{$\begin{array}{c}\text { Estatura-para-idad } \\
\text { (escore-z) }\end{array}$} \\
\hline & $\mathbf{N}$ & $(\%)$ & $\mathbf{m}$ & $(\mathrm{dp})$ & $\mathbf{m}$ & $(d p)$ \\
\hline \multicolumn{7}{|l|}{ Início do pré-natal } \\
\hline Até $3^{\circ}$ mês de gestação & 61 & $(91,0)$ & $-0,07$ & $(0,86)$ & 0,58 & $(1,35)$ \\
\hline Após $3^{\circ}$ mês & 6 & $(9,0)$ & $-1,15$ & $(1,82)$ & 0,61 & $(1,02)$ \\
\hline p-valor ${ }^{\mathrm{b}}$ & & & \multicolumn{2}{|c|}{0,01} & \multicolumn{2}{|c|}{0,96} \\
\hline \multicolumn{7}{|l|}{ No de consultas pré-natal ${ }^{a}$} \\
\hline$<6$ consultas & 14 & $(20,9)$ & $-0,58$ & $(1,50)$ & 0,54 & $(1,25)$ \\
\hline$\geq 6$ consultas & 52 & $(77,6)$ & $-0,04$ & $(0,82)$ & 0,61 & $(1,36)$ \\
\hline p-valor ${ }^{\mathrm{b}}$ & & & & 0,21 & & 0,87 \\
\hline \multicolumn{7}{|l|}{ Tipo de parto } \\
\hline Vaginal & 42 & $(62,7)$ & $-0,30$ & $(1,13)$ & 0,46 & $(1,08)$ \\
\hline Cesárea & 25 & $(37,3)$ & 0,05 & $(0,73)$ & 0,79 & $(1,65)$ \\
\hline p-valor & & & \multicolumn{2}{|c|}{0,18} & \multicolumn{2}{|c|}{0,34} \\
\hline \multicolumn{7}{|l|}{ Nasceu no Brasil } \\
\hline Sim & 60 & $(89,6)$ & $-0,14$ & $(0,86)$ & 0,58 & $(1,31)$ \\
\hline Não & 7 & $(10,4)$ & $-0,38$ & $(1,97)$ & 0,59 & $(1,52)$ \\
\hline p-valor ${ }^{\mathrm{b}}$ & & & \multicolumn{2}{|c|}{0,76} & \multicolumn{2}{|c|}{0,98} \\
\hline \multicolumn{7}{|l|}{ Sexo da criança } \\
\hline Masculino & 23 & $(34,3)$ & $-0,52$ & $(0,84)$ & 0,48 & $(1,76)$ \\
\hline Feminino & 44 & $(65,7)$ & 0,02 & $(1,05)$ & 0,64 & $(1,04)$ \\
\hline p-valor & & & \multicolumn{2}{|c|}{0,04} & \multicolumn{2}{|c|}{0,65} \\
\hline \multicolumn{7}{|l|}{ Peso ao nascer (gramas) ${ }^{\mathrm{a}}$} \\
\hline$\geq 2500 \mathrm{~g}$ & 52 & $(77,6)$ & $-0,04$ & $(0,85)$ & 0,63 & $(1,09)$ \\
\hline$<2500 \mathrm{~g}$ & 7 & $(10,4)$ & $-0,50$ & $(0,90)$ & 0,36 & $(2,77)$ \\
\hline p-valor ${ }^{\mathrm{b}}$ & & & & 0,19 & & 0,80 \\
\hline \multicolumn{7}{|l|}{ Idade da criança } \\
\hline$\leq 12$ meses & 30 & $(44,8)$ & $-0,24$ & $(0,82)$ & 1,06 & $(1,49)$ \\
\hline $12 \mathrm{a} \leq 24$ meses & 19 & $(28,4)$ & $-0,46$ & $(1,46)$ & 0,39 & $(1,00)$ \\
\hline$>24$ meses & 18 & $(26,9)$ & 0,26 & $(0,51)$ & 0,00 & $(1,07)$ \\
\hline p-valor ${ }^{\mathrm{b}}$ & & & \multicolumn{2}{|c|}{0,08} & \multicolumn{2}{|c|}{0,02} \\
\hline \multicolumn{7}{|c|}{ Cuidado diário da criança (diurno) } \\
\hline Creche & 28 & $(41,8)$ & $-0,12$ & $(0,83)$ & $-0,08$ & $(0,80)$ \\
\hline Mãe & 28 & $(41,8)$ & $-0,29$ & $(1,18)$ & 0,73 & $(1,09)$ \\
\hline Outros & 11 & $(16,4)$ & $-0,03$ & $(1,00)$ & 1,90 & $(1,83)$ \\
\hline p-valor ${ }^{\mathrm{b}}$ & & & & & & \\
\hline Acompanhamento em esta & & & & & & \\
\hline Sim & 63 & $(94,0)$ & $-0,19$ & $(1,03)$ & 0,59 & $(1,36)$ \\
\hline Não & 4 & $(6,0)$ & 0,23 & $(0,16)$ & 0,55 & $(0,68)$ \\
\hline p-valor ${ }^{\mathrm{b}}$ & & & & & & \\
\hline Tempo de aleitamento ma & & & & & & \\
\hline$<3$ meses & 28 & $(41,9)$ & $-0,20$ & $(0,73)$ & 0,85 & $(1,60)$ \\
\hline$\geq 3 \mathrm{e}<6$ meses & 25 & $(37,3)$ & $-0,35$ & $(1,31)$ & 0,07 & $(1,07)$ \\
\hline$\geq 6$ meses & 7 & $(10,4)$ & 0,17 & $(0,94)$ & 0,88 & $(0,75)$ \\
\hline Ainda amamenta & 7 & $(10,4)$ & 0,28 & $(0,69)$ & 1,07 & $(0,91)$ \\
\hline p-valor ${ }^{\mathrm{b}}$ & & & & 0,38 & & 0,10 \\
\hline Hospitalização & & & & & & \\
\hline Sim & 14 & $(20,9)$ & 0,16 & $(0,74)$ & 0,30 & $(0,63)$ \\
\hline Não & 53 & $(79,1)$ & $-0,25$ & $(1,06)$ & 0,66 & $(1,44)$ \\
\hline p-valor ${ }^{b}$ & & & & & & \\
\hline
\end{tabular}


de residência no país, simultaneamente, apenas o tempo de residência manteve-se associado à utilização de serviços de saúde ${ }^{27}$.

Dentre as mulheres entrevistadas, aproximadamente metade residia há mais de um ano no mesmo domicílio. O maior tempo de permanência em determinado local tende a fortalecer a rede de apoio do morador, principalmente no que se refere à saúde ${ }^{28,29}$. Além disso, um importante fator para o acesso da assistência à saúde é a proximidade do domicílio com uma unidade de saúde, sendo que neste estudo, 55,2\% dos bairros onde as entrevistadas residiam, possuíam algum tipo de estabelecimento de saúde vinculado à Atenção Básica.

No entanto, ao investigar as visitas das equipes de saúde no último ano, $88,1 \%$ das entrevistadas referiram nunca ter recebido visita de um membro da equipe de saúde. De acordo com a Política Nacional de Atenção Básica, a visita à família deve ocorrer em média uma vez por mês, considerando que as famílias que possuem crianças, idosos e doentes crônicos devem ser visitadas mais vezes ${ }^{30}$. Essas visitas possibilitam intervenções plausíveis e precoces, de forma que atenda às particularidades de cada realidade familiar, minimizando, assim, o risco de intercorrências no processo de crescimento e desenvolvimento infantil. Ao analisar a cobertura da ESF quanto aos bairros no qual as entrevistadas residiam, observou-se que 55,2\% dos bairros possuíam Unidade Básica de Saúde (UBS) implantada no referido bairro ${ }^{31}$. No entanto, não foi possível avaliar se os bairros que possuíam UBS possuíam também equipe de ESF completa para atender a população daquela região e, ainda, se a área de cobertura de cada unidade abrangia as residências dessas famílias.

A inserção no mercado de trabalho pode melhorar o poder de compra da população, influenciando positivamente na qualidade de vida e saúde da família. No entanto, neste estudo, mais da metade das entrevistadas encontrava-se desempregada e $43 \%$ das famílias avaliadas apresentaram renda per capita inferior a um quarto de salário mínimo, o que representa expressivo quadro de vulnerabilidade econômica e social. Apesar de não ter sido verificado diferença significativa quanto aos indicadores antropométricos, tal condição pode influenciar diretamente a saúde da criança, principalmente na garantia de alimentação adequada ${ }^{32}$. Em países de baixa e média renda, o trabalho materno é necessário como fonte de renda familiar suplementar, no entanto, o pouco investimento social e a infra-estrutura precária para o cuidado com a criança pode acarretar em prejuízos para sua saúde e bem-estar ${ }^{33}$.

Segundo Biscegli et al. ${ }^{34}$, nos casos em que a situação familiar alcança a extrema pobreza, a creche tem papel fundamental, pois, é nesse espaço educativo que a criança acaba por realizar dois terços de suas refeições diárias suprindo, assim, a maioria das necessidades básicas de nutrientes das crianças matriculadas. No entanto, a estatura para idade do grupo pesquisado apresentou diferença significativa entre os grupos, sendo identificada menor média entre as crianças que eram cuidadas diariamente em creches, quando comparadas às que eram cuidadas pelas mães, corroborando a revisão sistemática realizada por Pedraza et al. ${ }^{35}$, a qual indica que crianças que frequentam creches estão mais propícias ao acometimento de distúrbios nutricionais, do que aquelas que não frequentam esse espaço. $\mathrm{O}$ cuidado com a criança, também está relacionado à carga horária de trabalho materna, a exemplo da associação do trabalho materno com hábitos alimentares inadequados na infância ${ }^{36}$, relação esta que também pode estar presente entre filhos de mulheres imigrantes, visto que estas mulheres, muitas vezes, migram em busca de inserção no mercado de trabalho do país de destino.

Quanto à associação da estatura para idade com número de moradores por cômodo, esse aspecto é utilizado na Atenção Básica em Saúde como um dos parâmetros para avaliação de risco social e de saúde, de forma a refletir o potencial de adoecimento da família, avaliada a partir da escala de risco familiar de Melo et al. ${ }^{37}$, os quais afirmam que famílias com o equivalente a um morador por cômodo pode indicar risco duas vezes maior e as que tem mais de um morador por cômodo pode ter risco três vezes maior em relação àquelas com menos de um morador por cômodo. Pode-se inferir então que, 32,8\% dos domicílios de haitianos residentes em Cuiabá apresentam potencial de adoecimento da família. Desta forma, faz-se necessário um olhar mais atento a esse grupo populacional, visto que, de acordo com a escala de risco familiar supracitada, estas crianças apresentariam maior risco de adoecimento.

A OMS preconiza que as consultas pré-natais sejam iniciadas anteriormente ao terceiro mês gestacional e, ainda, que sejam realizadas ao menos seis consultas durante a gestação ${ }^{38}$. Dentre as entrevistadas, 64,2\% relataram que residiam em Cuiabá desde o início da gestação e, entre estas, 91,0\% iniciaram o acompanhamento pré-natal até o terceiro mês gestacional, o que pode ter 
influenciado no indicador de peso ao nascer relativamente melhor, quando comparados ao verificados no Haiti, sendo a prevalência de baixo peso ao nascer observada neste estudo $(10,4 \%)$, menos da metade do estimado no Haiti no ano de 2009 (25\%) e superior aos 8,0\% estimado para o Brasil no mesmo período ${ }^{39}$. Quanto ao índice de IMC para idade, verificou-se maior média deste indicador entre crianças com mães que iniciaram o pré-natal antes do terceiro mês gestacional, quando comparadas àquelas que iniciaram após este período.

Dentre as crianças avaliadas, $89,6 \%$ nasceram em território nacional, ou seja, deveriam ser acompanhadas no Brasil desde o nascimento. Para acompanhar adequadamente o crescimento e o desenvolvimento da criança, o Ministério da Saúde ${ }^{2}$ recomenda que no primeiro ano de vida sejam realizadas ao menos sete consultas com profissionais de saúde, sendo reduzidas para duas no segundo ano e anuais a partir daí. Dentre as crianças avaliadas, $44,8 \%$ tinham até um ano de idade, necessitando assim de um acompanhamento periódico por parte dos profissionais de saúde. No âmbito geral, $94,0 \%$ da totalidade das crianças avaliadas neste estudo foram, de algum modo, atendidas por profissionais de saúde, apesar de não ser possível identificar a periodicidade e a qualidade do acompanhamento com base nos dados coletados.

Quanto ao AME, apesar de não ter sido verificada diferença significativa com os indicadores antropométricos, ressalta-se que $41,9 \%$ das entrevistadas referiram tempo de AME inferior a três meses de vida da criança, o que pode comprometer sua saúde por longo período, visto que o AME tem a função não apenas de suprir as necessidades nutricionais, mas também de proteção, como redução do risco de alergias, desenvolvimento da cavidade bucal, diminuição do risco de diabetes e obesidade, sendo preconizado até o sexto mês de vida ${ }^{40}$. Para avaliação do aleitamento materno neste grupo deve-se considerar a prática realizada pelas mulheres haitianas em seu país de origem, isso porque no Haiti, o aleitamento materno, de forma geral, não costuma alcançar (ou seguir) as recomendações da OMS, sendo estimado que em 2012 que apenas $40,0 \%$ das crianças até cinco meses de vida eram amentadas no Haiti. Em contrapartida, $17,0 \%$ das crianças entre seis e nove meses avaliadas no presente estudo não eram alimentadas adequadamente por estarem apenas em aleitamento materno, e dessa maneira, não recebendo nutrientes necessários para essa etapa da vida ${ }^{41}$, apesar de não poder ser taxativo em classificar tal prática como cultural, esse modo de amamentação pode ser seguido pelas mulheres haitianas mesmo fora dos seu país de origem, necessitando que recebam orientações mais eficazes.

A hospitalização, por sua vez, pode fragilizar ainda mais o quadro de saúde infantil, isso porque o ambiente hospitalar, além de estranho para a criança, é repleto de novas possibilidades de infecções ${ }^{42}$. Quanto ao histórico de hospitalização, 20,9\% das crianças avaliadas já estiveram internadas em unidades hospitalares em algum período desde seu nascimento.

Destacam-se como principais limitações deste estudo a inexistência de registros oficiais do atual local de residência das crianças, o que leva a dificuldade de encontrar os possíveis participantes; falta de acesso a algumas cadernetas de vacinações; e dificuldade de comunicação, sendo necessário o tradutor para intermediar o diálogo. Devido ao pequeno tamanho amostral, houve reduzida precisão das estimativas apresentadas, contribuindo para diminuir o poder do estudo. Ressalta-se também a expressiva mobilidade da população haitiana no Brasil, que é usualmente gerida pela oferta de mercado de trabalho. Inicialmente, na capital mato-grossense estes imigrantes foram absorvidos pelo mercado de construção civil $^{14}$ norteado pelas obras da Copa do Mundo FIFA. Após esse período, a diminuição da oferta de trabalhos neste setor pode ter motivado a busca por oportunidades de trabalhos diversificados e cada vez mais escassos, o que pode ter inviabilizado a avaliação de todas as crianças nascidas em Cuiabá-MT. Embora o número seja consideravelmente baixo, foram avaliadas todas as crianças encontradas, equivalendo a aproximadamente $70,0 \%$ dos registros de nascimentos de filhos de mulheres haitianas em Cuiabá entre 2013 e 2015.

Os resultados do presente estudo possibilitam conhecer o estado nutricional, o perfil socioeconômico e as condições básicas de saúde das crianças descendentes de mulheres haitianas em Cuiabá-MT. A maioria das crianças nasceu no Brasil e apenas duas foram classificadas com IMC abaixo do adequado para a idade, sendo observada associação das maiores médias de IMC para idade com início do pré-natal até o terceiro mês gestacional e o sexo feminino e da estatura para idade com o cuidado diário da criança e número de moradores por cômodo. Faz-se necessário atentar-se para as condições socioeconômicas precárias nas quais estas crianças estão inseridas, visto que são fatores que apresentam impacto direto no estado nutricional no início da infância. 


\section{Colaboradores}

DRR Batista e AP Muraro participaram na concepção do estudo, coleta, análise e interpretação dos dados, além da redação do artigo e revisão crítica. PRM Rodrigues e AM Souza contribuíram para a discussão dos resultados e revisaram criticamente o artigo. R Sichieri contribuiu com a revisão crítica da análise dos dados e redação do artigo com contribuição intelectual relevante. Todos os autores aprovaram a versão final do artigo e são responsáveis por todos os aspectos do trabalho.

\section{Agradecimentos}

Os autores são extremamente gratos à Coordenação do Centro de Pastoral para Migrantes de Cuiabá, às mulheres e crianças que aceitaram participar do inquérito, aos entrevistadores bilíngues e aos alunos de graduação e pós-graduação em Saúde Coletiva envolvidos na coleta e digitação dos dados.

Agradecemos pelo financiamento à Fundação de Amparo à Pesquisa do Estado de Mato Grosso (FAPEMAT) e ao Conselho Nacional de Desenvolvimento Científico e Tecnológico (CNPq).

\section{Referências}

1. Sociedade Brasileira de Pediatria (SBP). Avaliação nutricional da criança e do adolescente: Manual de Orientação. São Paulo: SBP; 2009.

2. Brasil. Ministério da Saúde (MS). Saúde da criança: crescimento e desenvolvimento. Brasília: MS; 2012.

3. World Health Organization (WHO). Caring for the child's healthy growth and development [internet]. 2015 [acessado 2016 Jul 5]. Disponível em: http://apps.who. int/iris/bitstream/10665/204356/1/9789241504997_ ParticipantsManual_eng.pdf?ua=1

4. Organización de las Naciones Unidas para la Alimentación y la Agricultura (FAO), Organización Panamericana de la Salud (OPAS). 2016 América Latina y el Caribe. Panorama de la seguridad alimentaria y nutricional. Sistemas alimentarios sostenibles para poner fin al hambre y la malnutrición. Santiago: OPAS; 2017.

5. Ayoya MA, Heidkamp R, Ngnie-Teta I, Pierre JM, Stoltzfus RJ. Child malnutrition in Haiti: progress despite disasters. Glob Saúde Sci Pract 2013; 1(3):389396.

6. Pimentel M, Cotinguiba GC. Wout, raketè, fwontyè, anpil mizè: reflexões sobre os limites da alteridade em relação à imigração haitiana para o Brasil. Universitas Relações Internacionais 2014; 12(1):73-86.

7. Faria AV. A diáspora haitiana para o Brasil: o novo fluxo migratório (2010-2012) [dissertação]. Belo Horizonte: Pontifícia Universidade Católica de Minas Gerais; 2013.

8. Faria AV, Fernandes D. Análise temporo-espacial e perfil da imigração haitiana no Brasil - 2010-2014. In: Anais do Seminário "Migrações Internacionais, Refúgio e Políticas”. São Paulo; 2016.

9. Cavalcanti L, Oliveira T, Araújo D. A inserção dos imigrantes no mercado de trabalho brasileiro. Relatório Anual 2016. Brasília: OBMigra; 2016.

10. Fernandes D, Castro MCG, Ribeiro C. Migração Haitiana para o Brasil: Minas Gerais como destino, a fala dos haitianos. In: Anais do XVI Seminário sobre Economia Mineira; 16-20 set 2014; Diamantina; Universidade Federal de Minas Gerais; 2014.

11. Batista DRR, Gugelmin AS, Muraro AP. Acompanhamento pré-natal de mulheres brasileiras e haitianas em Mato Grosso. Rev Bras Saude Materno Infantil 2018; 18(2):327-336.

12. Batista DRR, Muraro AP. Características maternas, assistenciais e dos recém-nascidos filhos de imigrantes haitianas em mato grosso entre 2013 e 2015. In: $7^{\circ}$ Congresso Brasileiro de Ciências Sociais e Humanas em Saúde. 09-12 out 2016. Cuiabá: Abrasco; 2016. p. 244.

13. Borges FT, Muraro AP, Leão LHC, Carvalho LA, Siqueira CEG. Socioeconomic and Health Profile of Haitian Immigrants in a Brazilian Amazon State. $J$ Immigr Minor Health 2018; 20(6):1373-1379.

14. Leão LHC, Muraro AP, Palos CC, Martins MAC, Borges FT. Migração internacional, saúde e trabalho: uma análise sobre os haitianos em Mato Grosso, Brasil. Cad Saude Publica 2017; 33(7):e0018181.

15. Araújo CLP. Avaliação Nutricional de Crianças. In: Kac G, Sichieri R, Gigante DP, organizadores. Epidemiologia nutricional. Rio de Janeiro: Editora Fiocruz, Atheneu; 2007. p. 49-63. 
16. World Health Organization (WHO). Child Growth Standards. Length/height-for-age, weight-for-age, weight-for-length, weight-for-height and body mass index-for-age. Methods and development. Geneva: WHO; 2006.

17. World Health Organization (WHO). Physical Status: the use and interpretation of anthropometry. WHO Expert Committee. Geneva: WHO; 1995.

18. Brito CJ, Grigoletto MES, Nóbrega OT, Córdova C. Dimensionamento de amostras e o mito dos números mágicos: ponto de vista. Rev Andal Med Deporte 2016; 9(1):29-31.

19. Luiz RR, Magnanini MF. A lógica da determinação do tamanho da amostra em investigações epidemiológicas. Cad Saúde Coletiva 2000. 8(2):9-28.

20. Felisbino-Mendes MS, Campos MD, Lana FCF. Avaliação do estado nutricional de crianças menores de 10 anos no município de Ferros, Minas Gerais. Rev Esc Enferm USP 2010; 44(2):257-265.

21. Cuiabá. Secretaria Municipal de Saúde. Atenção Básica. Unidades de Saúde [internet]. 2017. [acessado 2017 Abr 22]. Disponível em: http://www.cuiaba.mt.gov. br/secretarias/saude/atencao-basica/

22. Menegaz A, Brunken GS, Espinosa MM, Guimarães LV, Lima-Lopes MA, Martins MSAS. Insegurança alimentar domiciliar, estado nutricional e morbidade referida de crianças residentes em municípios da região de expansão agrícola em Mato Grosso. In: Guimarães LV, Pignatti MG, Souza DP, organizadores. Saúde Coletiva: múltiplos olhares em pesquisa. Cuiabá: EdUFMT; 2012. p. 259-276.

23. Joint LA. Sistema educacional e desigualdades sociais no Haiti: o caso das escolas católicas. Pro-Posições 2008; 19(56):181-191.

24. Fernandes D, Castro MCG. Relatório do Projeto "Estudos sobre migração haitiana ao Brasil e diálogo bilateral” [internet]. 2014. [acessado 2017 Abr 25]. Dsponível em: https://obs.org.br/cooperacao/746-projeto-estudos-sobre-a-migracao-haitiana-ao-brasil-e-dialogobilateral

25. Padilha B. Saúde dos imigrantes: multidimensionalidade, desigualdades e acessibilidade em Portugal. Rev Inter Mob Hum 2013; 21(40):49-68.

26. Silveira C, Carneiro Junior N, Ribeiro MCSA, Barata RCB. Living conditions and access to health services by Bolivian immigrants in the city of São Paulo, Brazil. Cad Saude Publica 2013; 29(10):2017-2027.

27. Alves JFS, Martins MAC, Borges FT, Silveira C, Muraro AP. Utilização de serviços de saúde por imigrantes haitianos na grande Cuiabá, Mato Grosso. Cien Saude Colet 2019; 24(12):4677-4686.

28. Pereira APS, Teixeira GM, Bressan CAB, Martini JG. O genograma e o ecomapa no cuidado de enfermagem em saúde da família. Rev Bras Enferm 2009; 62(3):407416.

29. Souza IP, Bellato R, Araújo LFS, Almeida KBB. Genograma e ecomapa como ferramentas para compreensão do cuidado familiar no adoecimento crônico de jovem. Texto Contexto Enferm. 2016; 25(4):1-10.

30. Brasil. Ministério da Saúde (MS). Política Nacional de Atenção Básica. Brasília: MS; 2012.
31. Souza MHN, Silveira GS, Pinto AFS, Sodré VRD, Ghelman LG. Avaliação do estado nutricional e da saúde de crianças e adolescentes na prática assistencial do enfermeiro. Cogitare Enferm 2013; 18(1):29-35.

32. Santos AMA, Tejada CAO, Ewerling F. Os determinantes socioeconômicos do estado de saúde das crianças do Brasil rural. Rev. Econ. Sociol. Rural 2012; 50(3):473-492.

33. Géa-Horta T, Felisbino-Mendes MS, Ortiz RJF, Velasquez-Melendez G. Association between maternal socioeconomic factors and nutritional outcomes in children under 5 years of age. J Pediatr 2016; 92(6):574-580.

34. Biscegli TS, Polis LB, Santos LM, Vicentin M. Avaliação do estado nutricional e do desenvolvimento neuropsicomotor em crianças frequentadoras de creche. Rev Paul Pediatr 2007; 25(4):337-342.

35. Pedraza DF, Souza MM, Rocha ACD. Fatores associados ao estado nutricional de crianças pré-escolares brasileiras assistidas em creches públicas: uma revisão sistemática. Rev Nutr 2015; 28(4):451-464.

36. Ducci AL, Vannuchi MTO, Souza SNDH, Tacla MTGM, Lima LS. Aleitamento materno e consumo alimentar de crianças menores de um ano em um município do Sul do Brasil. Rev Bras Pesq Saúde 2013; 15(1):49-58.

37. Melo RHV, Vilar RLA, Ferreira AF, Pereira EJS, Carneiro NEA, Freitas NGHB, Diniz Júnior J. Análise de risco familiar na Estratégia Saúde da Família: uma vivência compartilhada entre preceptores, discentes e agentes comunitários de saúde. Revista Brasileira de Inovação Tecnológica em Saúde 2013; 3(4):58-71.

38. World Health Organization (WHO). New guidelines on antenatal care for a positive pregnancy experience. Sexual and reproductive health. Geneva: WHO; 2016. [acessado 2016 Dez 29]. Disponível em: http://www. who.int/reproductivehealth/news/antenatal-care/en/

39. World Health Organization (WHO). World Health Statistics. Geneva: WHO; 2011. [acessado 2016 Dez 29]. Disponível em: http://www.who.int/whosis/ whostat/EN_WHS2011_Full.pdf

40. Brasil. Ministério da Saúde (MS). Saúde da criança: aleitamento materno e alimentação complementar. $2^{\mathrm{a}}$ ed. Brasília: MS; 2015.

41. République D’Haiti. Ministère de la Santé Publique et de la Population (MSPP). Enquête Mortalité, Morbidité et Utilisation des Services. EMMUS-V. Haïti 2012. Porto Príncipe: MSPP; 2013.

42. Carvalho ES, Marques SR. Infecção hospitalar em pediatria. Jornal de Pediatria 1999; 75(Supl. 1):S31-35.

Artigo apresentado em 11/07/2018

Aprovado em 26/11/2018

Versão final apresentada em 28/11/2018 